\title{
Reconhecimento de Instrumentos de Percussão e Criação Automática de Arquivo Multipista
}

\author{
Lindokeny R. S. Moura* Dr. José Maria P. de Menezes Jr.** \\ * Curso de Engenharia Elétrica, Universidade Federal do Piauí, PI, \\ (e-mail: lindokeny@gmail.com). \\ ** Curso de Engenharia Elétrica, Universidade Federal do Piauí, PI \\ (e-mail: josemenezesjr@ufpi.edu.br)
}

\begin{abstract}
Musical editing is usually limited when musical instruments are put together into a single audio track. Therefore, it is a matter of interest to identify mixed sounds and separate them into their respective tracks. This article proposes the automation of this process, more precisely, it aims to work with percussion instruments, isolating them in distinct tracks without the need of a musician or musical producer for such task. To achieve this, two approaches are used: the first proposal uses digital filters, while the second involves machine learning concepts through the parameterization of audio stretches. In addition to recognizing the existence of a certain instrument in the mixed audio, the present work identifies the time instants in which the sound of such an instrument occurs, thus allowing both the creation of distinct tracks as well as the choice of substitute instruments (samples).

Resumo: A edição musical é normalmente limitada quando instrumentos musicais estão juntos em um único canal de áudio. Desta forma, é interessante identificar sons misturados e separá-los em seus respectivos canais. Este artigo propõe a automatização deste processo, mais precisamente, procura trabalhar com instrumentos de percussão, isolando-os em canais distintos sem a necessidade de um músico ou produtor musical para tal tarefa. Para isso duas abordagens são utilizadas: a primeira proposta utiliza filtros digitais enquanto a segunda envolve os conceitos de aprendizagem de máquinas através da parametrização de trechos do áudio. Além de reconhecer a existência de determinado instrumento no áudio misturado, o presente trabalho identifica os instantes em que o som de tal instrumento ocorre, logo sendo possível tanto a criação de canais distintos como também a escolha de instrumentos substitutos (samples).
\end{abstract}

\section{Keywords: Machine Learning; Signal processing; Pattern Recognition; Digital Filters; Audio Parametrization. \\ Palavras-chaves: Aprendizagem de Máquinas; Reconhecimento de Padrões; Processamento de sinais; Filtros Digitais; Parametrização de áudio.}

\section{INTRODUÇÃO}

O sistema auditivo humano é capaz de realizar a importante tarefa de identificar entre várias fontes sonoras superpostas aquela que lhe for mais conveniente (Sofianos, 2012). No entanto, a capacidade do aparelho auditivo distinguir sons misturados não se limita à conversação. Quando se observa uma música, apesar da voz está combinada com vários instrumentos musicais, geralmente se percebe com facilidade a letra da canção. Verifica-se ainda que ouvidos mais treinados, como os dos músicos, conseguem diferenciar até mesmo instrumentos musicais (Nwe and Li, 2008).

Por outro lado, as máquinas ainda não conseguem separar de maneira completa sinais de áudio previamente somados tal como os humanos (Sofianos, 2012). Assim, para se produzir uma música de karaokê, por exemplo, é necessária a presença de um profissional para escutar, memorizar e gravar cada instrumento presente na música original. Este processo demanda tempo mesmo para pequenas canções, logo, é de grande valia uma ferramenta computacional que reconheça automaticamente instrumentos em uma música.

Dentre vários instrumentos da música popular ocidental a bateria é a responsável pela ênfase e forma do ritmo. A possibilidade da máquina analisar a bateria permite a automação de uma variedade de tarefas relacionadas ao processamento de sinais de áudio (Wu et al., 2018). Por esse motivo uma das técnicas do presente trabalho trata do reconhecimento a partir da filtragem digital dos principais instrumentos que compõe a bateria: bumbo, caixa e chimbal. Alternativamente, a outra abordagem aqui empregada é capaz de identificar um arranjo musical formado por dois ou três sons de percussão quaisquer e convertê-los para as peças da bateria citadas anteriormente, utilizando-se reconhecimento de padrões.

Uma notável revisão sobre o reconhecimento de bateria, ou transcrição automática de bateria (ADT), é feita por $\mathrm{Wu}$ et al. (2018). Nesse trabalho constata-se que poucos au- 
tores propuseram algoritmos para essa linha de pesquisa. Souza et al. (2015) classificam sons isolados das peças metálicas da bateria e conseguem boa taxa de acerto com sua proposta que utiliza características temporais aliada a uma técnica espectral, porém, segundo análise de $\mathrm{Wu}$ et al. (2018), quando o classificador é aplicado a áudios do mundo real, a performance cai drasticamente. Por outro lado, o presente artigo contribui para o reconhecimento de áudios do mundo real.

O restante deste artigo está estruturado do seguinte modo: a seção 2 inicia o desenvolvimento a partir da definição de conceitos básicos importantes para o entendimento do trabalho. Já na seção 3 é mostrado o desenvolvimento das duas técnicas propostas de reconhecimento, portanto aqui há a ligação entre a fundamentação teórica e os resultados presentes na seção 4, onde é possível ouvir os áudios deste trabalho. Por fim, após avaliar o desempenho das técnicas propostas, a seção 5 apresenta as considerações finais, vantagens e limitações das propostas, bem como um direcionamento para próximos trabalhos.

\section{FUNDAMENTAÇÃO TEÓRICA}

Para melhor entendimento deste artigo é interessante a apresentação sucinta de algumas características do som e sua representação no domínio da frequência, destacando-se as peculiaridades dos instrumentos a serem identificados. Detecção de extremos, filtragem digital, reconhecimento de padrões e extração de características são também subseções desta fundamentação teórica.

\subsection{Características do som}

O som possui algumas características que muitas vezes facilitam a identificação da sua fonte sem precisar vê-la. De acordo com Resende (2014) suas principais propriedades estão listadas a seguir.

(1) Altura: classifica o som em grave, médio ou agudo.

(2) Intensidade: relaciona o volume ou amplitude do som como fraco ou forte.

(3) Timbre: qualidade que permite diferenciar um som de outro.

Entre as características citadas o timbre pode ser considerado o mais relevante na diferenciação de sons superpostos, mais detalhes podem ser encontrados em Allen (2018).

\subsection{Espectros dos sinais de áudio}

A representação dos sinais de áudio no domínio da frequência pode ser encontrada utilizando-se a transformada discreta de Fourier (DFT), resumida em (1), em que $f$ é o sinal de entrada com tamanho $N$ e a saída $F$ é um sinal de mesmo tamanho representando as amplitudes das componentes cosseno e seno, ou seja, o espectro de frequência.

$$
F(n)=\sum_{k=0}^{N-1} f(k) e^{-j 2 \pi \frac{k n}{N}} .
$$

O cálculo da DFT via (1) envolve muitas multiplicações e adições, entretanto o algoritmo da transformada rápida de Fourier (FFT) pode reduzir a quantidade de cálculos com base na linearidade do processo (Lathi and Green, 2005).

\subsection{Principais instrumentos da bateria}

Apesar da bateria ser composta por pelo menos oito instrumentos, apenas três formam a base da maioria das músicas populares ocidentais, sendo eles bumbo, caixa e chimbal. As outras peças como tons e demais pratos normalmente são requisitadas nos enfeites conhecidos por frases de bateria. Deste modo, a seguir são descritas as características no tempo e na frequência dos principais instrumentos que compõe a bateria.

(1) O bumbo, maior peça da bateria, tem uma sonoridade grave e de curta duração. Possui energia com realce nas frequências abaixo de $200 \mathrm{~Hz}$.

(2) A caixa apresenta um som mais impactante, também de curta duração. Geralmente suas frequências mais destacadas situam-se a partir de $300 \mathrm{~Hz}$, região de média frequência.

(3) O chimbal tem seu som conduzido por um pedal no qual pode resultar em som de curta ou longa duração, conhecidos respectivamente por chimbal fechado e chimbal aberto. Sua resposta em frequência cobre praticamente todo o espectro audível, tal como o ruído branco. Assim, diferentemente de bumbo e caixa, além das regiões grave e médio, no chimbal há informação frequencial na região aguda.

\subsection{Detecção de extremos}

Assim como no reconhecimento de voz, o processo de identificação de instrumentos tem seu início na segmentação do áudio em trechos de interesse. Alguns trabalhos, como o de Chougule and Shah (2018), utilizam os conceitos de energia do sinal e taxa de cruzamento por zero para realizar a detecção de extremos. O presente artigo propõe uma metodologia mais simples, aqui aplica-se a variância do próprio sinal, dada por (2); onde $\sigma^{2}$ representa a variância, $n$ o número de amostras, $X_{k}$ a amostra atual e $\bar{X}$ a média aritmética.

$$
\sigma^{2}=\sum_{k=1}^{n} \frac{\left(X_{k}-\bar{X}\right)^{2}}{n-1} .
$$

Na metodologia proposta poderá ser verificado os benefícios da aplicação da variância.

\subsection{Filtragem digital}

Utiliza-se a filtragem quando se deseja mudar ou eliminar as amplitudes dos componentes em frequência de um sinal. O filtro pode ser do tipo passa-baixas, passa-altas, passa-faixa ou rejeita-faixa. Tipicamente, filtros digitais são categorizados como resposta infinita ao impulso (IIR) ou resposta finita ao impulso (FIR), o primeiro é descrito por equações de diferença de maneira recursiva, enquanto o segundo é não recursivo.

Lathi and Green (2005) afirmam que os filtros digitais FIR são sempre estáveis, flexíveis, apresentam resposta de fase linear e são relativamente fáceis de serem implementados, quando comparados com os filtros IIR. Qualidades como essas tornam o tipo FIR a escolha mais popular entre os projetistas de filtros digitais, fato que justifica a presença desse tipo de filtro no presente trabalho. 


\subsection{Reconhecimento de padrões}

O reconhecimento de padrões envolve, essencialmente, as seguintes etapas de processamento: pré-processamanto, extração de parâmetros e classificação. O pré-processamanto, tem o objetivo de eliminar dados desnecessários fazendo com que a entrada apresente apenas dados relevantes para o reconhecimento do objeto em análise. A extração de parâmetros, também denominada etapa de seleção de atributos, consiste na análise dos dados de entrada a fim de extrair e derivar informações úteis para o processo de reconhecimento. O estágio final do reconhecimento de padrões é a classificação, ou etapa de tomada de decisão, que analisa as características extraídas dos dados de entrada e declara o objeto em análise como pertencente a uma determinada categoria (Matos, 2008).

Sistemas automáticos de aprendizado podem ser do tipo supervisionado, em que geralmente há a necessidade de especialista humano, ou do tipo não supervisionado, no qual se identifica subconjuntos de dados que compartilham certas similaridades (Real, 2014). Os algoritmos mais difundidos para os dois tipos de aprendizado são respectivamente o K-Nearest Neighbors (K-NN) e o K-Means (KMédias). Este último se resume em, dado um número $\mathrm{K}$ de centros desejáveis, onde estes $\mathrm{K}$ centros são escolhidos arbitrariamente na inicialização, calcular e recalcular os melhores $\mathrm{K}$ centros no conjunto de características de forma que estes fiquem distribuídos o mais homogêneo possível em seus grupos.

\subsection{Extração de características}

Silva et al. (2018) em seu trabalho de comparação forense de locutor conceituam a extração de características como a transformação do sinal de áudio em um conjunto de vetores, igualmente espaçados no tempo, capazes de descrever uma característica presente no sinal de voz. Instrumentos de percussão também podem ser parametrizados, como por exemplo no trabalho de Souza et al. (2015), em que consideram a extração de características a etapa responsável por alterar a representação dos dados originais em um conjunto de características mais representativas para os algoritmos de aprendizagem de máquina.

Verifica-se em alguns trabalhos relacionados ao reconhecimento de voz a utilização de características advindas de componentes cepstrais, em especial o MFCC (Mel Frequency Cepstral Coefficient), como em Lima et al. (2017) e Barbosa and Silva (2015). Apesar de muitas vezes o MFCC ser considerado o estado da arte no que diz respeito à descrição de áudio, Souza et al. (2015) incentivam a comparação em cenários semelhantes com a técnica LSF (Linear Spectral Frequencies). Esta última é uma versão alternativa da representação dos coeficientes LPC (Linear Predictive Coding), em que um sinal pode ser representado como a combinação linear das amostras previamente observadas, conforme (3):

$$
\widehat{x}_{k}=\sum_{i=1}^{p} a_{i} x_{k-i}
$$

onde $k$ é o índice atual e $p$ a ordem dos coeficientes $a_{i}$. Estes podem ser calculados com auxílio de algum algoritmo que minimize o erro de predição (Souza et al., 2015).

\section{METODOLOGIA PROPOSTA}

O primeiro passo para o reconhecimento do bumbo, caixa e chimbal em áudios com sinais superpostos se dá na detecção de extremos, ou seja, na segmentação do arquivo em trechos de interesse. Essa segmentação, como dito na fundamentação teórica, pode ser feita através da variância do próprio sinal da seguinte forma: a cada $n$ amostras calcula-se a variância por (2), resumindo a informação de $n$ para 1 amostra. Ao final deste processo obtém-se uma espécie de envelope superior que acompanha a dinâmica do sinal original, porém $n$ vezes menor.

É visto na Figura 1 um áudio com frequência de amostragem 44,1 kHz, o sinal gerado a partir de sua variância tomada a cada 500 amostras e a segmentação automática em trechos de interesse. Os extremos dos trechos são obtidos por uma heurística que leva em conta os picos do sinal de variância para definir o ponto inicial e final de cada trecho importante. Além do sinal da variância ser 500 vezes menor que o áudio original, tornando rápida a detecção de picos, percebe-se na Figura 1 que onde não há áudio determinístico a variância aproxima-se de zero. Essa vantagem estatística permite uma correta segmentação de trechos mesmo em áudios ruidosos.

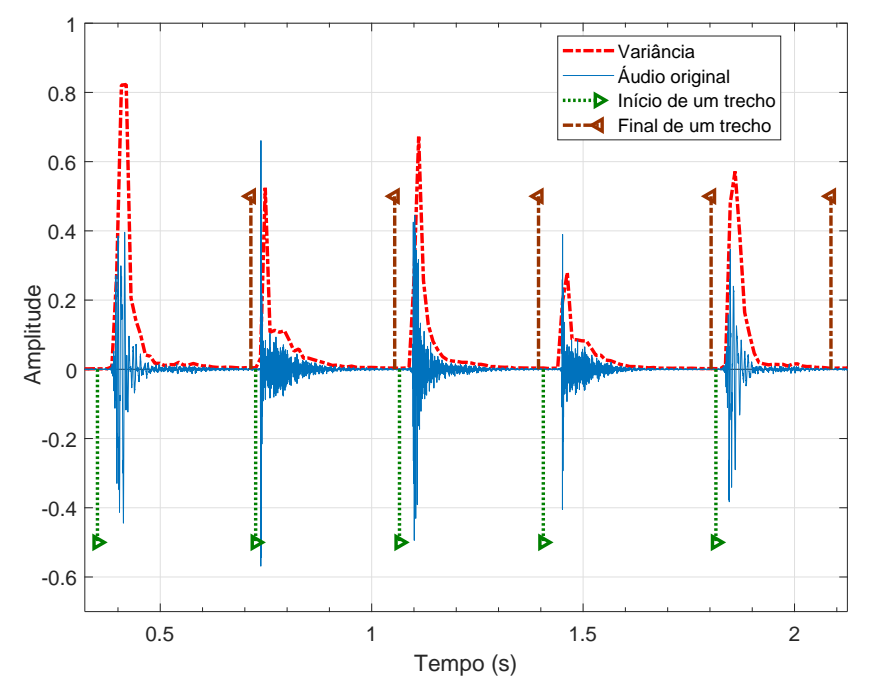

Figura 1. Exemplo de áudio, sinal gerado a partir da variância e extremos detectados automaticamente.

A segmentação prévia do áudio de maneira automática para o reconhecimento de instrumentos de percussão, neste artigo, é utilizada nas duas técnicas propostas.

\subsection{Reconhecimento por filtragem digital}

É possível localizar os instantes em que um determinado instrumento ocorre utilizando-se um filtro passafaixa FIR de ordem elevada, com frequências de corte inferior e superior compreendendo a região de máxima 
frequência desse instrumento. Como o desafio está na localização das frequências de cortes de maneira automática, implementou-se a rotina a seguir com base na abordagem de sistemas especialistas e do conhecimento das frequências dos instrumentos.

Um laço seleciona trecho a trecho e os envia para outra função que analisa o espectro e retorna as frequências que definem a banda de maior energia do trecho em questão, ou seja, as possíveis frequências de corte inferior e superior do filtro passa-faixa para bumbo e para caixa. Se a frequência de corte inferior for menor que $150 \mathrm{~Hz}$, muito provavelmente o trecho analisado contém um bumbo; se as frequências de corte inferior e superior estiverem entre 165 e $500 \mathrm{~Hz}$, é a vez da presença da caixa. O laço tem seu fim a partir do momento em que são encontradas as frequências de corte para os dois instrumentos.

Depois de encontradas as frequências de corte para esses instrumentos, filtra-se o áudio original utilizando-se os respectivos valores de corte e em seguida detectam-se os picos do sinal resultante. Os locais de picos em cada sinal filtrado são, portanto, os instantes em que bumbo e caixa aparecem.

Por não possuir uma região de destaque na frequência, para detectar o chimbal atribui-se ao filtro uma frequência extremamente alta $(10$ e $22 \mathrm{kHz}$ ), pois é desejado que não se realce as ocorrências de bumbo ou caixa. Após filtrar o sinal original e detectar os picos, deve-se fazer o tratamento desse instrumento, isto é, separar locais de chimbal aberto e fechado. Esse procedimento pode ser feito automaticamente definindo-se como local de chimbal aberto os casos em que há decaimento lento após o pico, e chimbal fechado quando o decaimento for rápido.

O último passo é a construção de até quatro canais distintos e escolha dos instrumentos substitutos, para bumbo, caixa, chimbal fechado e chimbal aberto.

\subsection{Reconhecimento por classificação de padrões}

A segunda proposta de reconhecimento de instrumentos de percussão é através da parametrização do áudio previamente segmentado, sendo este um arranjo musical com até três tipos de sons percussivos quaisquer. A ideia é analisar cada batida presente nos segmentos de áudio e converte-la em som de bateria. Devido a abrangência do sinal de entrada, o reconhecimento aqui se dá de forma não supervisionada, isto é, não existe banco de dados previamente rotulado, há apenas uma nuvem de dados em que o algoritmo K-Means deve clusterizar.

Com relação a escolha dos atributos, levando-se em conta os apontamentos de Souza et al. (2015), verifica-se que tanto a técnica LPC quanto MFCC retornam parâmetros determinantes para classificação de instrumentos de percussão, no entanto, o LPC fornece os melhores resultados com poucos coeficientes. Outros dois atributos foram escolhidos empiricamente com base na separabilidade visual dos dados no espaço. Assim, a parametrização de cada trecho é feita por três atributos distintos: o primeiro é advindo dá técnica LPC com ordem 1, o segundo é obtido a partir da variância do espectro de frequência, já o último é simplesmente o valor da maior frequência (frequência fundamental).
Ao fim da parametrização do último trecho, normalizamse os dados para que os atributos tenham a mesma ordem de grandeza, posteriormente o K-Means decide o grupo em que cada trecho pertence. Após o agrupamento, para evitar inversões dos instrumentos, padroniza-se como grupo do bumbo aquele que possui o trecho cujo atributo de frequência fundamental é o menor (grave). Havendo três tipos de batida, identifica-se como grupo do chimbal aquele onde está presente o trecho cujo atributo de frequência fundamental é o maior (agudo). Em todo caso, o grupo da caixa é o que não foi automaticamente rotulado.

\subsection{Construção de canais distintos}

Antes de tudo, este trabalho não tem interesse em recuperar necessariamente o mesmo som da bateria reconhecida. Com base no trabalho de Caceres (2012), acredita-se que é mais interessante utilizar nos novos canais sons substitutos proveniente de instrumentos com maior qualidade e/ou gravados em melhores condições. Áudios desse tipo são conhecidos como samples e tem seu uso bastante difundido entre profissionais da música.

Sabendo disso e de que essa etapa é comum para as duas propostas de reconhecimento, a criação de novos canais se dá a partir de uma matriz de zeros $c \times N$, na qual $c$ representa o canal do instrumento reconhecido e $N$ o número de amostras do arquivo original.

Nessa matriz são inseridos samples apenas nos locais de pico do instrumento reconhecido e em sua devida linha, de maneira que entre um som e outro os dados permaneçam em zero, as vantagens desses períodos de silêncio entre os samples serão discutidas nos resultados.

\section{RESULTADOS}

Esta seção tem o propósito de avaliar o desempenho das técnicas de reconhecimento dos instrumentos de percussão discutidas neste trabalho, filtros digitais e reconhecimento de padrões não supervisionado. Observe a seguir os detalhes dos áudios de teste.

(1) Áudio 1: trecho da música "cansei de esperar" (bonde do forró), composta por diversos instrumentos musicais, incluindo voz.

Ouça no link https://bit.ly/2UV7yp9

(2) Áudio 2: áudio gravado a partir do microfone do notebook com três tipos de sons distintos, sendo o primeiro e segundo gerados por tipos diferentes de batidas na carcaça do computador, enquanto o terceiro som foi produzido por um recurso de beatbox.

Ouça no link https://bit.ly/2WqsWmq

O áudio 1 pode ser testado com a proposta de filtros digitais, pois além de outros instrumentos, nele estão presentes as peças bumbo, caixa e chimbal a serem reconhecidas. Já o áudio 2 deve ser analisado pela técnica de reconhecimento de padrões não supervisionado, pois esse áudio contém três tipos de sons de percussão formando um arranjo musical que se substituído por samples de bateria, soaria melhor.

\section{1 Áudio 1}

Após a segmentação do áudio e análise dos trechos verificou-se que o bumbo do áudio 1 retorna máxima 
frequência entre 56,5 e $72 \mathrm{~Hz}$; já para a caixa os valores são entre 258,4 e $269 \mathrm{~Hz}$. Filtrando o áudio 1 com essas frequências de corte e utilizando a frequência atribuída na metodologia para o chimbal, obtem-se os sinais filtrados presentes na parte inferior da Figura 2.
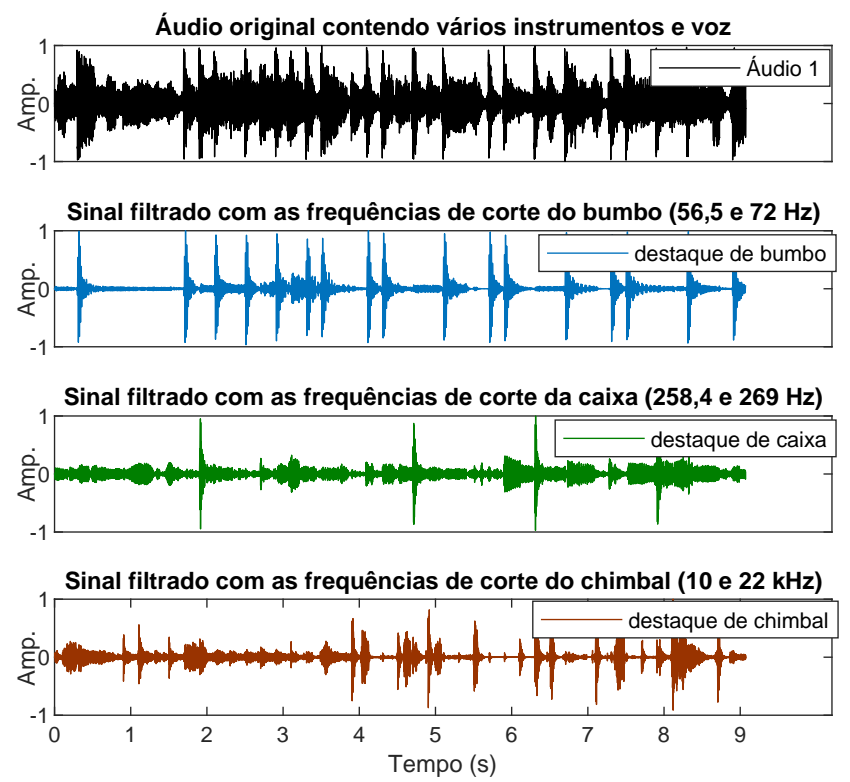

Figura 2. Sinais filtrados em suas respectivas frequências de corte.

Criando-se novos canais e inserindo samples apenas nos locais de picos dos sinais filtrados, obtem-se o resultado final do reconhecimento via filtros digitais para áudio 1 presente na Figura 3, em que a parte superior é o arquivo original e as outras a representação dos canais dedicados aos instrumentos reconhecidos. Percebe-se que há momentos de silêncio nos novos canais que não são observados no arquivo original, o porquê disso é a superposição no áudio 1 de outros instrumentos como guitarra, baixo, sanfona e até mesmo a voz, enquanto nos novos canais construídos só estão presentes as principais peças da bateria.

A vantagem dos momentos de silêncio é evidenciada na edição musical, por exemplo, é possível amplificar o sinal de um dos instrumentos sem o risco de aumentar o ruído, pois o vetor do novo áudio é preenchido por zeros entre um som e outro. Paralelamente, é possível equalizar cada instrumento sem interferir nos outros, uma vez que os samples são inseridos em canais dedicados.

Mesmo reconhecendo uma música com muitos instrumentos, o resultado é aceitável, pois há sincronismo dos instrumentos e a única falha observada é a não detecção do chimbal fechado em três ocasiões. Assim, a técnica de filtros digitais foi capaz de acertar todos os 17 bumbos, todas as 4 caixas, o único chimbal aberto e 6 das 9 ocasiões de chimbal fechado, resultando em 90,3\% de acerto.

O resultado do reconhecimento do áudio 1 pode ser verificado no link a seguir:

https://bit.ly/2WldnfI
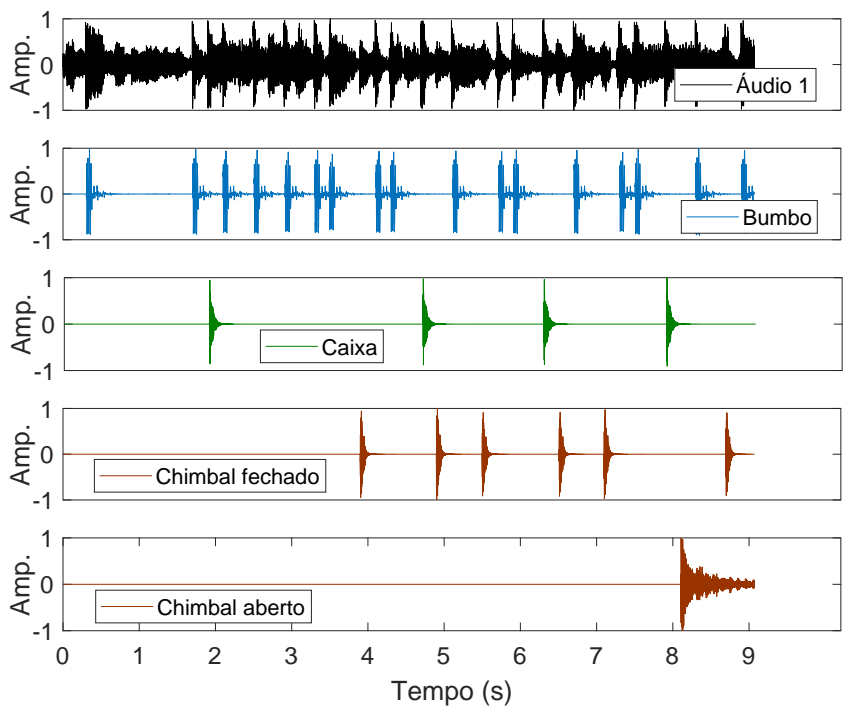

Figura 3. Audio 1 (original) e instrumentos reconhecidos

O sincronismo do resultado com relação ao áudio original pode ser verificado neste outro link:

https://bit.ly/2HJExcO

\section{2 Áudio 2}

Após a segmentação do áudio 2, os 22 trechos produziram 22 conjuntos de 3 parâmetros, conforme descrito na subseção 3.2. Esses dados podem ser visualizados no espaço tridimensional na Figura 4 e é evidente que os dados de cada tipo de som são geograficamente separáveis, logo, supõe-se que algoritmo K-Means não tem dificuldades em agrupar os 3 conjuntos. Por fim, segue a construção dos canais com base na sequência de identificação dos trechos.

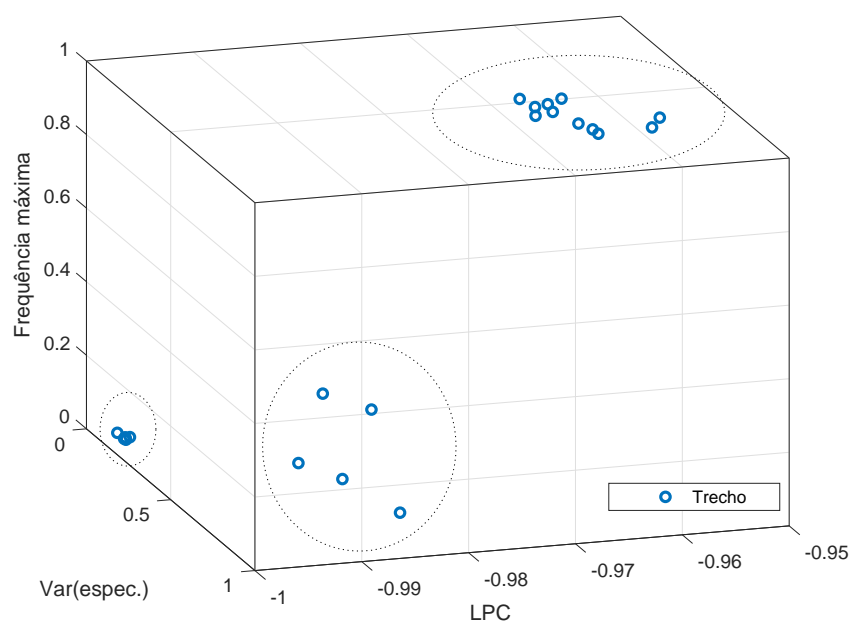

Figura 4. Nuvem de dados do áudio 2.

A Figura 5 contém o resultado do reconhecimento pela classificação de padrões, na qual a parte superior é o áudio original e na inferior o áudio convertido para bateria. Verifica-se que os novos canais estão sincronizados com 
o arquivo original, além disso, não há erro de reconhecimento, o que resulta em $100 \%$ de acerto.

O resultado da conversão das batidas do áudio 2 em peças da bateria pode ser verificado no link a seguir:

https://bit.ly/2FzJLEx

O sincronismo do resultado com relação ao áudio 2 pode ser verificado neste outro link:

https://bit.ly/2HJPIT7
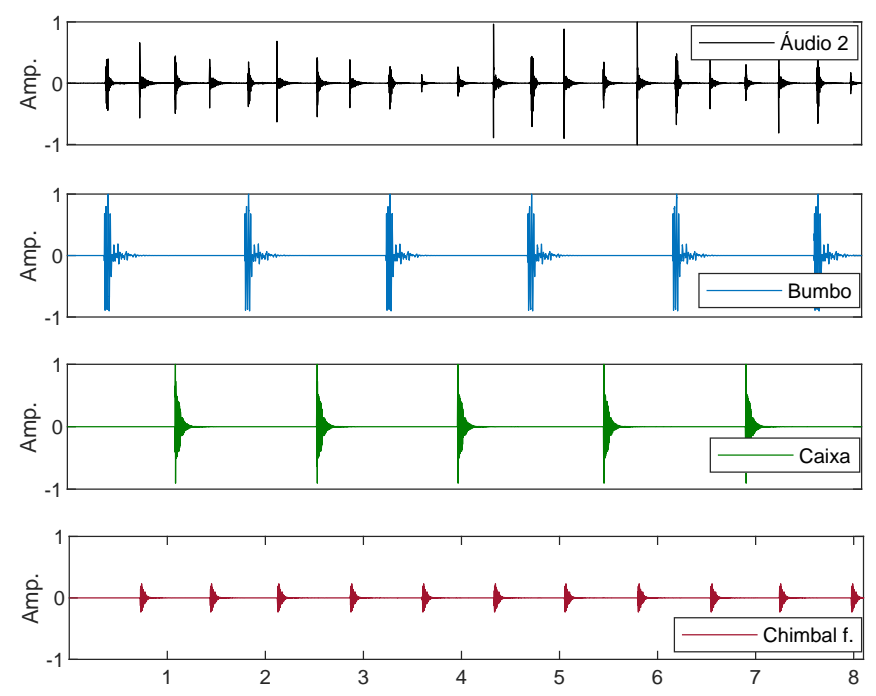

Figura 5. Áudio 2 (original) e instrumentos reconhecidos.

\section{CONCLUSÕES}

Os resultados obtidos na seção 4 mostram que é possível o computador reconhecer automaticamente instrumentos de percussão em áudios misturados e em seguida criar novos canais de áudio dedicados para os principais instrumentos da bateria.

Verifica-se que a técnica que utiliza filtros digitais é eficaz no que diz respeito ao reconhecimento, pois mesmo com muitos sinais superpostos o filtro consegue destacar os instantes do bumbo e da caixa. Houve uma certa limitação em relação ao chimbal, mas é esperado, uma vez que esse instrumento não possui uma frequência de destaque.

De modo similar, o reconhecimento via classificação de padrões consegue converter/substituir arranjos musicais de outras formas de percussão nos principais instrumentos da bateria. O fato dessa técnica ser não supervisionada traz mais uma vantagem: não limita o timbre do áudio de entrada, ou seja, é possível gravar arranjos com até três tipos de sons de fontes diversas e ter o arranjo musical equivalente formado por bumbo, caixa e chimbal.

Como trabalho futuro, para a primeira proposta esperase o aprofundamento de técnicas auxiliares que possam melhorar a identificação do chimbal. Em classificação de padrões, deseja-se tanto aumentar a quantidade de tipos de sons de entrada, como também reconhecer batidas simultâneas.

\section{REFERÊNCIAS}

Allen, E.J. (2018). Perception and Processing of Pitch and Timbre in Human Cortex. Master's thesis, Faculty of the University of Minnesotaa.

Barbosa, F.G. and Silva, W.L.S. (2015). Reconhecimento de voz para autenticação biométrica utilizando máquinas de vetores de suporte e os coeficientes mel-cepstrais. In XII Simpósio Brasileiro de Automação Inteligente (SBAI). Natal - RN.

Caceres, G.T.S. (2012). When the levee breaks: A trajetória de uma gravação do microfone ao sampler. In $3^{\circ}$ Encontro Internacional de Música e Arte Sonora. Juiz de Fora - MG. URL http://www.ufjf .br/anais_ eimas/files/2012/02/Caceres.pdf.

Chougule, U.V. and Shah, S.K. (2018). Parameter based voice activity detection. In International Journal of Innovations \& Advancement in Computer Sciences (IJIACS), 7(3), 420-424.

Lathi, B.P. and Green, R.A. (2005). Linear systems and signals, volume 2. Oxford University Press New York.

Lima, P., Silva, W., and Barros, A. (2017). Análise de configurações de redes neurais para sistema de reconhecimento de padrões de sinais de voz utilizando parâmetros tcd de baixa ordem. In XIII Simpósio Brasileiro de Automação Inteligente (SBAI). Porto Alegre - RS.

Matos, F.M.S. (2008). Reconhecimento de faces utilizando a transformada cosseno discreta. Master's thesis, Universidade Federal da Paraíba.

Nwe, T.L. and Li, H. (2008). On fusion of timbremotivated features for singing voice detection and singer identification. In 2008 IEEE International Conference on Acoustics, Speech and Signal Processing, 2225-2228. doi:10.1109/ICASSP.2008.4518087.

Real, E.M. (2014). Investigação de Algoritmos Sequenciais de Agrupamento com Pré-Processamento de Dados em Aprendizado de Máquina. Master's thesis, Faculdade Campo Limpo Paulista.

Resende, D.D. (2014). Um modelo de Classificação Supervisionada com Rotulagem Automática para Reconhecimento de Audio de Web Rádios. Master's thesis, Universidade Federal de Ouro Preto.

Silva, A.P., Vieira, M.N., Barbosa, A.V., and Paiva, C.U.N. (2018). Avaliação de descritores acústicos utilizando estatística mvkd aplicada a comparação forense de locutor. In XXII Congresso Brasileiro de Automática (CBA). doi:10.20906/CPS/CBA2018-0155.

Sofianos, S. (2012). Singing Voice Extraction from Stereophonic Recordings. Ph.D. thesis, University of Hertfordshire, Hatfield.

Souza, V.M.A., Batista, G.E.A.P.A., and Souza-Filho, N.E. (2015). Automatic classification of drum sounds with indefinite pitch. In 2015 International Joint Conference on Neural Networks (IJCNN), 1-8. doi:10.1109/ IJCNN.2015.7280342.

Wu, C., Dittmar, C., Southall, C., Vogl, R., Widmer, G., Hockman, J., Müller, M., and Lerch, A. (2018). A review of automatic drum transcription. IEEE/ACM Transactions on Audio, Speech, and Language Processing, 26(9), 1457-1483.

\section{Apêndice A. LINK CONTENDO TODOS OS ÁUDIOS}

https://bit.ly/2H56MBl 